\title{
The role of social networks in students' learning experiences
}

\author{
Ilaria Liccardi \\ School of Electronics and \\ Computer Science \\ B32 University Road \\ University of Southampton \\ Hampshire, UK SO171BJ \\ il05r@ecs.soton.ac.uk \\ Elizabeth Massey \\ Faculty of Technology \\ Brayford Pool \\ University of Lincoln \\ Lincolnshire, UK LN6 7TS \\ bmassey@lincoln.ac.uk
}

\author{
Asma Ounnas \\ School of Electronics and \\ Computer Science \\ B32 University Road \\ University of Southampton \\ Hampshire, UK SO171BJ \\ ao05r@ecs.soton.ac.uk
}

Päivi Kinnunen
Department of Computer
Science and Engineering
Helsinki University of
Technology
Helsinki, 02015, Finland

\author{
Reena Pau \\ School of Electronics and \\ Computer Science \\ B32 University Road \\ University of Southampton \\ Hampshire, UK SO171BJ \\ rp05r@ecs.soton.ac.uk
}

Sarah Lewthwaite
Learning Science Research
Institute

School of Education

University of Nottingham

Nottingham, NG7 2RD, UK

ttxsem@nottingham.ac.uk

\author{
Marie-Anne Midy \\ School of EECS \\ Oregon State University \\ 1147 Kelley Engr. Ctr \\ Corvallis, OR 97330 , USA \\ midym@onid.orst.edu
}

\author{
Chandan Sarkar \\ School of EECS \\ Oregon State University \\ 1147 Kelley Engr. Ctr \\ Corvallis, OR 97330, USA \\ sarkar@eecs.oregonstate.edu
}

\begin{abstract}
The aim of this research is to investigate the role of social networks in computer science education. The Internet shows great potential for enhancing collaboration between people and the role of social software has become increasingly relevant in recent years. This research focuses on analyzing the role that social networks play in students' learning experiences. The construction of students' social networks, the evolution of these networks, and their effects on the students' learning experience in a university environment are examined.
\end{abstract}

\section{Categories and Subject Descriptors}

K.3.2 [Computer and Education]: Computer and Information Science Education - Computer science education

\section{General Terms}

Human Factors, Theory

\section{Keywords}

Social network, computer science education, student experience, higher education, learning process

\section{INTRODUCTION}

Identifying the right people to effectively resolve a problem or collaborate with others is a challenging task. There are clear advantages to getting this right, as it may involve people with varying levels of expertise working together collectively to resolve problems. A vast array of systems exist which employ users' stored profile data, identifying matches for collaboration. Social interaction within an online framework can help university students share experiences and collaborate on relevant topics. As such, social networks can act as a pedagogical agent, for example, with problem-based learning.

This paper is a literature review of social networks in education including both technical and social aspects of computersupported collaborative learning. The aim of this review is to highlight the complexity of the field of social learning and to bring forward some central aspects that need more investigation. This paper is a snapshot of an ongoing larger study. Based on this literature review, we will later gather empirical data to further uncover how social networks are formed and what makes them work efficiently.

As various possibilities for web-based education are investigated, researchers and universities are trying to provide effective web-based courses to students within the university, and as a mode of delivery for distance learners.

This paper focuses on pedagogy from a student perspective, concentrating on the computer science culture. Initially, the paper gives an overview of existing social software applications falling under the "Web 2.0" category, and discusses the potential for employing these applications in education. Particular attention will then be given to the perception of social networks and their current influence on the computer science students' learning experience. The paper also discusses the gap between the fast developing social software and its use for education. Because the paper is an effort by 
PhD students, educators can benefit from realizing the importance and influence of social networks on the students' academic lives, and their learning experience in particular, from a student perspective.

To understand the impact social networks have on the learning experience, it is worth a bit of discussion on how social networks are formed, how universities group students, and how students go about grouping themselves. Issues such as diversity and perceived value of social networks to students are also considered in this context. Examples of how the study of social networks can assist educators with their teaching - such as detecting plagiarism, forming groups for collaborative learning, enhancing distance learning, and building strong communities of experts - are given in different sections of the paper.

Considerable resources have been dedicated to establishing effective methods for locating people suitable for working groups. In higher education, universities frequently group students together to improve student performance. Some success has been attained in the use of web-based courses using shared spaces and annotation of web-pages, and adaptive desktops for accessing their evolving contents. Weblogs, wikis, logs and online documentation are also gaining popularity and pedagogic credence as part of the learning process, as is the evolution of associated collaborative communities.

Section 2 introduces the concept of social networks, presenting the ways in which networks can be formed, along with other types of group in which people can be aggregated. Section 3 describes current theories about how people learn. In section 4, the social aspects of collaborative learning are examined. Section 5 analyses the different social networks present in education, how they are formed, the influence of universities and diversity of this formation, their effect on the learning experience and communication within a social network. Section 6 gives an overview of computer-supported social network applications. Finally, Section 7 discusses the conclusions of this review.

\section{SOCIAL NETWORKS}

The study of social networks has received significant interest from researchers in various domains such as psychology, philosophy, education, and lately computer science - particularly in the field of artificial intelligence. This section defines what we mean by social networks, the way in which these networks form and evolve in our daily lives, and their relations to other types of group.

\subsection{Definition of Social Network}

Social networks are a social structure of nodes that represent individuals (or organizations) and the relationships between them within a certain domain. Therefore, social networks are usually built based on the strength of relationships and trust between the members (nodes). Scrutiny of the ways in which these nodes are connected has resulted in the identification of varying types of ties between nodes. In [2] and [33], the authors discuss the notion of describing connections in terms of strong and weak ties, or formal and informal relationships, respectively. In this context, a strong tie is one established directly between two people in the same network, whereas a weak tie is a relationship between two people connected through another person (two levels of separation).

\subsection{Algorithms}

Forming connections is a natural human process. Current research outlines the algorithms that model the resulting networks. Examples of these algorithms are investigated in graph theory [38] and have highlighted the social values and evolution of trust that occurs among the members of the network.

Constructing networks: The way in which people meet and form social networks in everyday life has caught the attention of many computer science researchers. The fact that we relate to and depend on our social network for such things as friendship, support, special interests and knowledge sharing has inspired algorithm developers to analyze these facets in a more abstract way. In [35] the authors describe the Neighboring Matchmaker methodology thus: two individuals who do not know each other are introduced by a mediator who is a friend of both and who can facilitate the creating of a new relationship. A similar method of creating or enlarging a network follows the Friend of a Friend concept, where the associations are inferred through mutual friends. However, with methods such as Friend of a Friend, trust and privacy issues become questionable due to the debatable measurement and inference of trust. Since social networks can serve as a rich resource of new knowledge and as a filter for identifying the information most relevant to our specific needs, a second naturally occurring networking method is Word of Mouth [37]. Here individuals obtain reviews, share knowledge and expertise, find experience and ask for advice or assistance from the neighboring individuals in their social network.

Searching networks: On searching for specific criteria within a social network, Zhang and Ackerman [101] studied the social characteristics of various searching algorithms that can be useful in finding individual features such as expertise, in order to understand the tradeoffs involved in the design of social network-based searching engines. The use of searching algorithms to navigate social networks can be highly beneficial in looking for a special individual and then identifying the people connected to her.

Network dynamics: People have been forming themselves into groups without the gratuitous nature of the internet or social software. Online communities are not new. So what is new about the interaction of people within online social networks? According to [53], what may be different is understanding the role social networks play in forming communities. Wellman views the difference between networks and groups: "Although people view the world in terms of groups they function in networks. In networked societies, boundaries are permeable, interactions are with diverse others, connections switch between networks, and hierarchies can be flatter and recursive." [91]. Due to the transparent and loosely-knit nature of social networks, members move in and out of communities without formalism. Indeed, according to [91], "Rather than fitting into the same group as those around them, each person has his/her own personal community." 
Inspired by the interactions within social networks, researchers use mathematical models to simulate what happens in daily life. Examples of algorithms in this context include coalition formation, network formation and stability, clustering algorithms, clubs algorithms, and game theory algorithms. Applications of these algorithms occur in a variety of fields include distributed processors, communication and computer networks, social economics, and multiplayer games. In education, these studies can be used in querying or visualizing the social networks of students in different areas such as assessment. For instance, if the teacher has a visualization of the network, then she can easily recognize plagiarism cases given that the students' friends and close colleagues are shown in the network. Then same thing applies in areas such as group formations. In some scenarios, a teacher may want to allocate the students to groups that do not already have their friends as participants. In this way, the groups will not suffer from cases where some students reduce their involvement, while their friends contribute more effort to compensate without complaining about their lazy friends.

\subsection{Social Network and other types of group}

There are many types of group that define different types of collaboration between individuals. According to the taxonomy of groups bellow, the most formal types of group are teams where individuals collaborate to deliver a specific and well-defined task. Within Computer Science, this can be construed as similar to Software Engineering project groups. Less formal types of group are determined as communities that demonstrate higher group cohesion and shared social values.

Communities: Also known as communities of knowledge, communities are informal groups of people that develop a shared way of working together to accomplish some activity [3]. The goal of the community is generally diverse even if the community has been formed to deal with a specific topic. The membership of a community is usually self-selected and self-organized in a similar manner to social networks.

There are various definitions for the spectrum of existing communities. As with the variations of groups in general, communities' categorization differ in relation to the following aspects:

- purpose of the community

- boundary of membership

- formalization of set-up

- formalization of co-ordination in terms of members' roles

- size of community

- composition of the community in terms of expertise

- frequency and type of interaction (whether face to face and/or via computer supported tools) [93] [3].

The most prominent group type among knowledge-sharing communities are the Communities of Practice (CoP). According to Wenger [92], CoP are groups of people who have a common interest in a subject, and collaborate to share ideas or find solutions. In theory CoPs provide a framework to approach studying and learning from a social standpoint. In an educational context social networks can be construed as CoP since they fulfill the criteria outlined by Wenger: social networks have a common goal, members contribute to the community, and members of the community have shared practices.

There are other types of community besides the CoP; for example, Communities of Interest (CoIs) and Communities of Commitment (CoCs) [21]. The variation of these types of community resides in the level of formality and contract value. Unlike $\mathrm{CoCs}$, CoPs have low formality and contract value, frequently articulated by the degree to which a community has to deliver concrete results. The classification of communities can be also based on their virtuality. A virtual community is one that has some form of computer system facilitating the communication between the members as a central element to its definition [67].

Networks: Besides social networks, another common form is Intentional Networks. Intentional networks, also known as "Networks of Practice" (NoPs), are an informal collection of collaborators selected to accomplish a specific task [97]. This grouping differs from teams since it is informal, has a shorter temporal duration, and low group cohesion. The members are not required to be familiar with each other as long as they can cooperate to deliver the task.

\section{LEARNING EXPERIENCE}

Learning is a complex phenomenon that can be approached from a variety of theoretical, historical and philosophical viewpoints. Different perspectives are called for to understand how people perceive knowledge construction, and to simultaneously comprehend the instructional process as a whole. For instance, phenomenographic studies provide understanding of 'inner worlds' of students and teachers (see e.g. [12], [51], [9], [43]). Activity theory [25], on the other hand, approaches phenomena from the holistic point of view. It aims at understanding the student learning process as a part of a larger context (see e.g. [9], [45]).

Design of educational model has been highly affected by Diana Laurillard's conversational framework [47] which states that within higher education, students' study may be mediated through the following interactions:

$$
\begin{aligned}
& \text { - } \operatorname{student(s)} \text { and student(s) } \\
& \text { - } \operatorname{student(s)} \text { and teacher(s) } \\
& \text { - } \operatorname{student(s)} \text { and the world. }
\end{aligned}
$$

Since social networks are generally considered well aligned with the pedagogies of social-cultural theories of learning, peer-to-peer relations and interactions are foremost in this study.

Säljö argues, "Access to the learner's perspective on the activities of teaching and learning is essential for understanding educational phenomena - and for improving education" (emphasis in original, [73]). This paper discusses how social networks can assist students to seek meaning in the context of their university studies. A central question within learning is 'how do students gain knowledge?' and phenomenog- 
raphy can help us to consider the complexity of this question by showing the different ways in which people experience and think about learning. The research of Marton et al. [51] highlights six distinct concepts of learning as:
1. increasing knowledge
2. memorizing and reproducing
3. applying
4. understanding
5. seeing something in a different way
6. changing as a person.

The first three concepts consider learning as primarily reproducing, while the latter three perceive learning as primarily seeking meaning. A social network that consists of many students may have some or all of the Marton's concepts of learning represented. There are however implications for the function of the network. This research aims to recognize the student as situated, and for this reason an understanding of the learning experience must be couched in terms of the functions and contradictions of the social network itself. There are clear benefits in applying the methodologies of activity theory to this end.

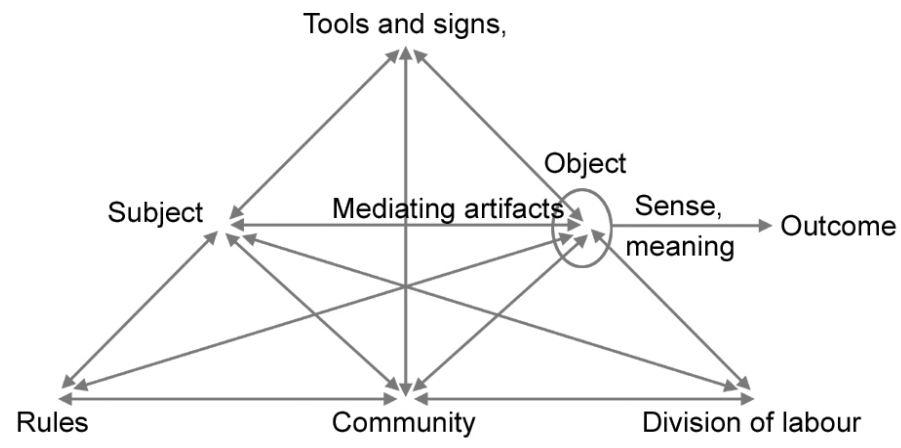

Figure 1. The structure of a human activity system[25]p. 78

Discussing learning from an activity theory point of view widens the concept of learning from an individual-centered concept, to a community level. The social network is conceived as an activity system (see figure 1) that constitutes subject (student) and object (knowledge construction). The subject's interaction with the object is further mediated by tools (e.g. computer, hardware and software) and a community that shares the same object, (the social network itself). To be able to interact with the community the relationship between the subject and community is mediated by rules. Division of labor, in turn, mediates the relationship between the community and object. From an activity theory standpoint, learning occurs during goal-directed activities as an expansion of one's scope of action.

\section{SOCIAL LEARNING}

The social dimension of learning has always been of great significance to both teachers and learners [6]. In [48], the authors argue that learning is a function of the activity, context and culture in which it occurs (situated learning theory), where social interaction is critical. The authors describe learning as a process: learners become involved in a "community of practice" which represents certain beliefs and behaviors, and, as a newcomer moves from the border of this community to its center, they become more active and engaged within the culture. Moreover, this theory claims that situated learning is usually unintentional rather than deliberate, so it is more effective for the learner to belong to a selfselecting community rather than to be assigned to a group. The situated learning concepts have descended from Vygotsky's social development theory, which also claims that the social interaction plays a fundamental role in the development of cognition [87]. In [90], Wegerif highlights the importance of the social side of learning when designing a course, more specifically in asynchronous learning networks. This paper shows that individual success or failure on the course may depend partly on the learner's feeling as either insider or outsider in the learning process.

In an educational context, small group activities have traditionally been used for their asserted benefit to student learning. However, small group activities frequently have a dual function at university, as students are subject to professional, as well as academic, pressures in the course of their studies. Following university, graduates can be faced with employers' demands for proficient team-working, communication, and project presentation. Small group activities are one way in which the educational system is trying to inculcate these skills and satisfy business demand. The small group dynamics is a much researched area (see [6], [84], [39], [40], [94]) providing many insights into the types of variable that affect a network's efficiency.

In an educational context, certain factors can notably promote or hinder group activities and outcomes. For instance, group composition may affect how efficiently a group achieves its set goals [14]. Therefore, it is optimal that there are both goal-oriented group members, and socially-oriented people within the same network. Borgatta and Bales [14] suggest that both are needed in order for a group/network to achieve its goals as well as experiencing the group as socially rewarding.

Shellens et al. [75] recently deprecated the importance of group composition in knowledge construction. They state that the impact of student characteristics can be of higher significance than characteristics of the discussion group students are allocated to. Task characteristics, individual learning styles and attitudes towards task-based learning are all cited as having a salient impact on learning outputs. Indeed, Shellens et al. [75] state that, within their research, no significant group characteristics were observed.

In addition to the composition of a group, several other significant factors influence how a group functions [60]. These include individual-based qualities such as motivation, enthusiasm, previous experience of group work, and communication abilities. Furthermore, participants' behavior, the students' attitudes, and encouragement from peers affect how the group works. Finally, group dynamics, the joint effort 
the students put in, and the quality of team-working, contribute to the way group works.

Problem-based learning (see for example, [76], [63]) provides a useful example of didactic activity that is based on smallgroup activity and is based heavily on the idea of social construction of knowledge. Here, learning is founded on solving problems based on real-life situations, interacting with peers and a facilitator. The interaction in a problem-based learning group differs greatly from the more passive transmission of information model found in traditional large-scale lectures. The emphasis is on active-learning: discussion, question asking, and sharing of knowledge with peers rather than passively listening or studying material that is selected by the teacher. The peer-to-peer support is a seminal positive aspect of problem-based learning that several studies have highlighted [10], [79], [60]. The awareness that peers are struggling with the same difficulties is important. The group also forms a network where one can seek help and assurance. This is regarded as beneficial, especially for students who do not have a large informal social network of friends.

The importance of the social aspect of learning is manifold, and bears further consideration. For example, Tinto [82] concludes that participation in a collaborative learning group enables students to develop a network of support. This is vital on many levels, particularly since a network helps a student to bond with broader social communities. A community of peers (network of support) thereby encourages a student's attendance and class participation. Furthermore, learning communities also give students the opportunity to meet both social and academic needs simultaneously. Tinto et al. (in [82]) stress that social affiliations serve as a vehicle through which academic involvement is engaged. This emphasis on the importance of the support provided by peers is seconded by Kinnunen and Malmi [41] amongst others.

Tinto [82] stresses that academic satisfaction is not enough for some students who suffer from social isolation. The lack of a social network can, together with other factors, result in such drastic measures as students dropping out of courses [42]. Conversely, sufficient social involvement can counterbalance a lack of academic involvement. This is especially important in the first year of study, after which academic issues tend to become more and more important.

\section{SOCIAL NETWORKS IN EDUCATION}

Typically, institutions use a range of various educational approaches in the classroom, tutorial, lab and lecture hall. Activities can take place face to face, but may also be mediated by social networking technologies include peer assessment, discussions, and collaborative work. Course designers have been quick to spot such opportunities [90] by way of chat rooms, discussion forums and collaborative work support tools which may be used in this way. The efficiency and effectiveness of such approaches are necessarily the subject of evaluation, analysis and debate [36].

The study of social networks within a learning domain encompasses the processes of social learning that occurs when a self-selecting group of people who have a common interest in a subject collaborate to share ideas or find solutions [77].
Observations of the processes and behaviors of self-selecting groups can be used to engineer interactions in groups orchestrated for specific educational purposes.

Social networking applications which incorporate Web 2.0 technologies demonstrate affordances, which could be available to utilize within the classroom. These operate with paradigms which are different to those observed within conventional e-learning tools. However utilizing social networking tools with large student groups might present problems. An advantage of increased awareness or appreciation of the complexity of typical observed behaviors in a social learning environment may enhance the academic's ability to manage the tools.

A recent study of the potential for semantic modeling of learners explores using Semantic Web-based social networks to facilitate the automatic and dynamic creation of students' networks within large online communities [66]. Enriching the semantics of network and membership descriptions can provide valuable information. This can be used to assist in tuning group allocations, enabling the network to be used for specific educational objectives.

\subsection{Social Network Formation}

Identifying the right personnel to effectively resolve a problem or collaborate with others is a challenging task, as stated at the beginning of this paper. A number of systems exist which aim to match personnel based on information provided in the users' profiles. Typically, such systems comprise an online form which must be completed [86] or a software agent which runs in the background on a computer to determine subjects the user is interested in [52] [32]. Determining factors include project roles, recorded publications and written communications with others.

Other systems help identify individuals with specific skills. Examples include agent-based expert finders [86], peerlearner finders [98], Neighbourhood Matchmaker method [35], or expertise recommenders [54]. In each of these systems, users' profile data is stored and, given a set of constraints, it is possible to locate partners. A group can then be formed from the resulting set of users. Agent-based expert finders [86] are optimized to find experts within a specific subject field. By noting expertise based on past work the system identifies the person who best matches the requested query.

The peer-learner finder [98] is a method that constructs a flexible and effective self-organization system. It works to group together similar learners according to their preferences and learning behaviors by employing a multi-agent mechanism which manages and organizes the learners and learner groups [35]. This method can be further extended with effective award and exchange algorithms that can cluster learners with similar preferences or interests into an individual community.

The Neighbourhood Matchmaker method provides a matchmaker as the middle link between two users. This aims to ensure that the matchmaker knows whether the users are suitable to work with one another. 
Finally, the Expertise Recommender assists in the identification of natural expertise [54]. Such methods are often employed in companies seeking to provide an identification service in which users can identify the key personnel needed and then form them into groups.

Other types of expert finder systems provide a set of preexisting groups for users to join. Projects such as StudioBRIDGE [99] allow people to locate colleagues with similar or identical interests. Tools such as Soari [54] enable the formation of links among friends, family, co-workers and acquaintances allowing attributes such as "weak" and "strong" ties. This method allows users to control how their information is distributed and shared. For example, parents may want to share their family photos only with people who are close friends or relatives [33].

In the context of computer science education two typical examples of social network formation are considered as examples. First a teacher selects a random set of students to accomplish a project task. Second, a set of students selfselect to form a group based on perceived expertise. We see then that social networks software can help to form teams by allowing users to identify appropriate groups of people to work and share their information with.

\subsection{Contextual influence on social network formation}

A central concern of this research are the influences that host institutions, departments and chosen curriculum can exert on the creation and deployment and of social networks for education, Each of these three influences could motivate or moderate the way in which educational social networks are developed and sustained. Typical instances of networks in this context include those directly sponsored by the university/department as part of a curriculum (curriculumspecific), and those spontaneously occurring established by students to complement their university work (curriculumrelated). In this respect, institutional influence is expressed both directly and indirectly. Engeström's model of Activity Theory [25] offers a useful tool with which to map explicitly such influences. To model this influence on curriculumspecific social networks 8 key factors could be considered:

1. The activity of interest, in this case social networking

2. The object or objective of activity: knowledge construction

3. The subject engaged in the activity: student

4. The tools mediating the activity: hardware, interface, learning platform, social software and delivery mechanisms

5. The rules and regulations mediating the activity: policies and pedagogic strategies

6. The division of labour mediating the activity: allocation of group roles

7. The community in which the activity is conducted: student group

8. The desired outcome towards which the activity is directed: collaborative learning.
Immediately, environmental influences (from the host institution/department) can be identified in several key areas. The hardware and software indirectly determines the technological affordances and constraints of the social networking software environment which they provide. These affordances and constraints can be construed as structural or embedded 'rules' that determine the limits of activity. For example, the usefulness of the network will be dependent on the provision of a network connection that may be high-speed, or uninterrupted. The available 'reach', or degree to which any member of a network can reach other members of the network, may be constrained or enhanced by institutional policy concerning agent-based expert finders (see 5.1). The environment also determines the available community and group membership, and this may impact on the 'division of labor', that is, the group roles that social network members subsequently adopt. Further to this, many computer science departments have introduced 'pair-programming', pairing students for assignments to reduce computer anxiety. If this pairing results in an unequal work-load, conflict that extends to social networks may occur, despite the perceived benefits for raising a student's self-efficacy and programming confidence.

Most obviously, the environment - via the university department - determines the curriculum content, and thus, the subject of discussion and pedagogical organization of the task. Finally, environmental influence is conveyed through explicit social rules that may take the form of Terms and Conditions of use, or other codes of practice. Understanding the environmental impact upon external, curriculum-related social networks is more difficult. These networks occur outside of the institution's direct sphere of influence, and whilst a network may be established to address curriculum content, by a student whose online identity is authenticated by the university, these two elements alone may be the only concrete evidence of institutional involvement.

However, students are situated. Multiple axes of influence bear upon a student at any one time, including institutional effects. A student may identify with, or internalize a university's values, thereby becoming an agent of the university within the social network. Examples of this agency include students' expressions of learning culture through their epistemologies, the value they place upon the resources and personnel they recruit, and the group roles they adopt to achieve their learning aims. Since students self-select their subject and institution, values commensurate with those of their institution can be expected.

\subsection{Diversity influence on social networks}

Diversity among university students is certain. Differences in ethnicity, class, language, gender, nationality, disability, capability and religion amongst students influence their communication within a particular network [55]. A diverse student body can either enhance or inhibit the student learning experience as defined in section 5.2. Studying for a computing degree is generally perceived to be a solitary existence, when in reality those that study computing rely on their social networks for help and support in their degree programs [50]. For example, a common feature of the computing curriculum is the lab culture. It is here where undergraduates experience a significant portion of their face- 
to-face interactions. The advantage of this type of environment, from a student's perspective, is the free access to a wide range of students in other year groups and other courses. This exposure can broaden a student's network to receive support and/or gain knowledge.

Self-efficacy theory claims that people's beliefs about their capabilities to realize a goal influences the way they approach tasks. Accordingly, students with strong self-efficacy have a higher level of confidence to access a social network, whereas those with weaker self-efficacy may not as they fear rejection from the network's established members. This level of inefficacy can exacerbate a student's sense of isolation [50], [8], creating a belief that no assistance is available for them. An example is the current drop-out rate of females in computer science courses. Cohoon states that these attrition rates occur due to a perceived lack of support from their academic environment [20]. This is also demonstrated by [22] who claim that females suffer from 'computer anxiety' (i.e. they get anxious using the computer in front of others). This can inhibit their overall contribution to the lab culture or group work assignments in class [50], [22].

Group work assignments, which are an inherent part of computing degrees, provide interesting challenges for students, in terms of communication, time management and knowledge. A diverse group of people bring together a range of issues. For example, international students not only grapple with the differences in their external environment, but also with the nuances of a foreign language. Differences, in both accent and meaning, can lead to misunderstandings in communication [62]. Misunderstandings can also arise through a clash of diverse personalities. Students are grouped in potentially random ways and in an effort to become a cohesive and productive unit, students must find alternative ways to resolve their differences [65].

An online social network has the potential to reduce social exclusion, thus increasing a student's self-efficacy. In the 1990s 'cyberspace' was initially deemed a place of escape from face-to-face essentialism, and 'the corporeal embodiment of gender and race' [7] with implications also for disabled people. Subsequent research suggests that the internet is a more controversial territory [88]. However, social networks do enable a different articulation of the self that allows a user to manage preconceptions. For example, a student who is a wheelchair user can control the disclosure of their disability online, deciding when, where, and if, their disability is relevant to a social network discussion. For some disabled students controlling disclosure in this way can facilitate social presence with potentially positive learning outcomes. However, it should be noted that for other students print impairments, such as dyslexia, may represent uncontrollable disclosure, with a negative impact on confidence and contributions. In these circumstances a student is disabled by the network, and both the student and network can suffer as a result.

Universally, students are diverse in their need to learn and do things in a particular way. This diverse blend of personalities can either mean conflict or agreement for group members (e.g. obsessive vs. lackadaisical). Conflicts caused by different personalities can have an adverse effect on the learning experience for individual members, and subsequently to the productivity of the social network [65]. Students need to adapt quickly to their environments in order for their learning experience to realize the full benefit that a social network can give.

\subsection{Social networks and learning experience}

Computer science is a discipline with its own distinct culture, and this affects the nature of social interaction online. As diversity is discussed elsewhere in this paper, the shared characteristics of computer science students with their study-mates and the impact of their shared tasks bear consideration. Prentice et al. distinguish between (real life) groups based around a common bond, and those based on a common identity [69]. A common bond group is based primarily on connections that exist among peers - for example in a friendship group. Attachment to a common identity group is based on an individual's identification with a group's purpose. Sassenburg [74] has subsequently established that the distinctions and processes outlined by Prentice et al. also apply to online groups.

In universities, entrance criteria are applied to an already self-selected population who have been educated to a similar level, in similar subjects and express similar interests. Thus, students' educational experiences at admission are broadly analogous. The vast majority of undergraduates have grown up in the same globalized technological milieu, and are characterized as a generation of 'millennials' or 'digital natives' [68], for whom computers and the internet are mundane, rather than exceptional. Computer science undergraduates are, by virtue of their specialization, highly computer literate and when forming study mates they can be expected to be initially based upon a common identity. Prentice et al. demonstrates that common identity groups show higher levels of conformity and adherence to group norms than common-bond groups [69]. This suggests that there will be limited diversity in common identity groups such as computer science. Sassenburg [74] has subsequently established that the distinctions and processes outlined by Prentice et al. also apply to online groups. This suggests that one might expect therefore that computer science's own cultural 'norms' would be magnified in curriculum-specific social networks. Rhode et al. [71] cite their own research and Tajfel [81] suggests that cultural norms in computer science can make processes of social identification more difficult and, therefore, successful community building less likely. To approach such generalizations in the case of computer scientists, this research must ensure close attention to the demographics of the student participants involved. The situated relationships between individuals, their social network and institution must be respected.

Computer science is a discipline with its own distinct culture, and this affects the nature of social interaction online. As diversity is discussed elsewhere in this paper, the shared characteristics of computer science students with their cohorts and the impact of their shared tasks bear consideration. 


\subsection{Communication in Social networks: a comparison of face-to-face and online per- spectives}

Communication, both physical and electronic, is part of everyday life. Communicating with colleagues about solutions to a current task assignment or making plans with friends for an evening's activities are simple daily interactions. These seemingly simple face-to-face interactions can, however, become complex when undertaken online, mainly because users have available technologies, such as email, short message service (SMS), and chat rooms, to overcome time and space constraints. Having a long discussion with a colleague who is not in the same work domain can be difficult. For example, using email infers that the response is delayed as it takes time to write a reply. Similarly using an SMS service can be difficult, due to bifurcations in conversation, the need to be allowed space to make their point, or a requirement to refer to previous conversations. Hence, online conversation can become convoluted as more users get involved.

Traditionally, study groups consist of a set of members, related by their selected course, who choose a quiet place to sit together and discuss the assigned classroom tasks. With the advent of on-line chat rooms, social software systems such as Facebook, and instant messenger systems, we come to the question of whether physical proximity is still of great importance to the educational process. Boulos et al. [15] noted that university student populations are more mobile and more diverse than ever. Wireless connectivity and egroups have allowed students to become members of collaborative online networks and study groups.

In Slattery's study [79] of students involved in learning communities, participants tended to form their own support groups that extended beyond the classroom, and spent more time together outside the class than did their colleagues in traditional stand-alone classes. "Students involved in the study saw those groups as critical to their ability to continue in college" [83]. In addition students acquired ownership of both their topic and the group, thus demonstrating "...an increased sense of responsibility to participate in the learning experience, and an awareness of their responsibility for both their learning and the learning of others." The evolution application of Web 2.0 technology such as in wikis, blogs and forums potentially make this process simpler and more natural. For example, blogs are employed as project lifecycle management tools as well as collaborative document building [16]. Additionally, since their introduction by Ward Cunningham in early 1995 [49], wikis have achieved sustained success. A variety of wiki engines are available and applied to a broad range of application domains [95]. Collaborative writing through the use of wikis is a vast and popular area for current research. Wikipedia [96] is one of the most successful and effective examples in collaborative writing, taking the form of an online encyclopedia which anyone can contribute to. Similarly, forums (e.g. Slashdot) are used to discuss topics of interest in a shared environment. As these collaborative technologies evolve continually toward a synchronous environment, a contributor (student) finds they are no longer working in isolation but are part of a much larger community.
Even with the ubiquity of Web 2.0 technologies, the literature suggests that text-based asynchronous ComputerMediated Communication (CMC) such as email, blogs and wikis do not have the capacity to support social and affective interaction since this form of CMC lacks the ability to process ambiguous content [72]. In comparison, face-to-face communication is considered to be the richest, and best for complex tasks. Research suggests that nonverbal cues increase speaker-listener interactions [56]. For example, an instructor can tell how well students are following the lesson based on facial expressions, body position, and eye contact.

Non-verbal cues also indicate emotions, feelings and mood, which each group member may or may not explicitly express. However, these are influential facets of a member's affective interactions. Garrison et al. [31] describes these as defining characteristics of social presence. Members of a social network who are not physically able to be present with the group and who are confined to on-line interactions must rely on symbolic representations of humor, feelings and mood, such as emoticons to experience a situation as if socially present [44], [34].

Communication in a social network is a crucial aspect of users' interactions. Studies show the main difference between CMC and face-to-face communication is the time it takes to accomplish the same task [13], [5]. Intuitively, since face-to-face communication provides both verbal and nonverbal feedback, groups can more quickly reach consensus [89], [4]. Research indicates that when the message content included jokes, expression of feelings, self-introductions, compliments, greetings and closures, experienced users rated CMC just as effective as face-to-face conversations. In summary, the literature indicates that for complex tasks involving decision making by multiple persons, students express a preference for face-to-face communication. Despite the distractions involved in group participation, social networks that manage their time effectively can supply a successful learning experience for each group member.

\section{COMPUTER-SUPPORTED SOCIAL NETWORK APPLICATIONS}

The emergence of the web as a collaborative and social environment has paved the way for a plethora of social networking software. Although this paper focuses on how social networks enhance the students' learning experience within the academic environment, it is useful to consider how social network software is also used "on the outside".

Tagging: Software which allows tagging of specific information such as del.icio.us [24] allows users to share their bookmarks. CiteUlike [18] and Flickr [28] both allow sharing of tagged pictures. FilmTrust [27] combines social networks with movie ratings by reading about movies, rating them, writing reviews and by maintaining lists of friends and ranking how much trust is given to these opinions.

Networking: Networking software has been developed to serve different purposes; the most common are: 1) to create and maintain the social network of either on-line or real-life friends, and 2) to re-unite past friends.

Software that allows users to maintain a social network of 
friends (both on-line "friends" and real-life mates) is very popular nowadays. Facebook [26] supports more than 30 million active users with an average growth rate of 3 percent weekly since January 2007 [17]. Facebook requires that members hold the status of "friend" to view information such as personal details, interest groups, messages from friends displayed on a 'wall'. Orkut [64] works much like Facebook and requires "friend" status to access information; however, text can be added to a user's wall by non-members. $M y S$ pace [61] is a compromise of the two by offering an interactive, user-submitted network of friends, personal profiles, blogs, groups, photos, music and videos. In MySpace one can browse other users' front pages; however, personal information is hidden from non-members.

Another popular software package Friends Reunited [29] allows users to find people who for whatever reason have lost touch, and would like to re-establish contact. Classmate [19] lets users locate those from their past allowing a wide selection of places and locations including business names, colleges, and military service. Classmate is linked with Canadian, French, German and Swedish sites. Similarly, friendster [30] and reunion [70] have the same purpose. These examples give a brief idea of the impact social software applications have on both local and global communities.

Co-authoring: Wikis allow users to collaborate online on shared ideas. The Wikipedia project [96] resembles an online encyclopedia which can be edited by anyone. There are provenance issues with software of this kind; however, the entries to the encyclopedia are regulated by 'senior wikipedia administrators' and other members of the community.

Sharing: Software which allows participants to share media through recommendation by providing similar items to one selected. Examples of video sharing include YouTube [100], veoh [85], dailymotion [23]. Software like LastFM [46] exists to recommend music based on prior choices. This tool allows participants to show and share lists with friends. Friends can be added through a common interest. For example, if a participant enjoys jazz music and is recommended a playlist of another participant they may become friends based on this common interest.

Simulated life on the web: This type of software provides the opportunity for users to interact online using their imagination and creativity. A demonstration of this is the virtual world Second Life [78], an immersive environment where members, using avatars, are able to explore, meet, enter into group activities, create content and trade with others online within a virtual economy with real world economic equivalence.

Technologies: These meta-applications enable social networking to take place. An example of this is Friend of a Friend, which is a semantic technique for connecting friends. This refers to the strength of a friendship. An advantage of an online social network is being able to search for people or interests, and a method for doing this is the use of folksonomies. A folksonomy is a way of categorizing web content (by the user). Users are able to develop their own tags and see each other's tags. An advantage of tagging is quicker retrieval of related content.

\subsection{Advantages}

Since the web is arguably better linked than the "real world", finding people on-line with shared interests is easier, and more likely. Nowadays many applications allow users to keep in touch with long term friends, family and to find new friends. In addition, new relationships based on the links between friends, and friends of friends are created. These new relationships are not limited to people users already know. Indeed, links are created in the act of stating an interest, or joining a network; in this action, users find other people who share the same opinions, hobbies, or university.

To maintain relationships, the computer-supported social network software provides various tools within the application (forums, tickets, online profiles, etc.). Thus users have more support options than when using one-to-one communications such as email. When a dedicated place is available for users to post specific comments, the opportunity to request information and gauge others' interactions creates available norms that can more easily be applied. Viewing others' comments and postings provides a double feedback to the user: first, they are using the right application at the correct place; secondly, other people have the same questions, interests, or ideas. This promotes a much needed sense of community. This reciprocating interaction applied to the university environment offers not only benefits to students but in the long term to the entire community.

\subsection{Disadvantages}

Links among individuals based on trust, affinity, and expertise versus friendship are not as well defined as in the real world. As the definition of strong and weak ties are vague in their application to online relationships, social software struggles to model and implement real world relationships. Online profiles can be a source of deception. Indeed, when a person mis-states their true identity or intentions, trust is broken, potentially negating the foundation of their online relationships.

It is much easier to lose contact online since online interaction is asynchronous communication. One party is invariably waiting for the other to reply. Conversely, interacting in close physical proximity exploits the non-verbal cues inherent in face-to-face communication. Naïve students over-reliant on online communication can be unwittingly cut off from the necessary communities with possibly disastrous academic consequences.

In terms of trust, the security of personal information online is increasingly important. Whilst the authenticity of online identities may be questioned, conversely the vulnerability of personal information online is generating increasing numbers of 'horror stories' covered in the mass media. Many social networking sites work on a basis of presumed trust, with users' profiles being displayed and available to registered users and guests (meaning non-registered users) by default, even if they do not belong to the same network or do not share the same interests. Progressively, many networks now give the users the facility to set their own level of disclosure, 
at a community level or to close friends only. However, this precaution is not yet available for all applications.

Negative consequences of sharing personal data in this way include spam to private email accounts, 'phishing' attempts at fraud and identity theft and vulnerability to malicious real-world activity aimed at damaging individuals or their property. Standards and policy for protecting user information is a vastly researched area, and beyond the scope of this paper. However, for users to fully benefit from online applications in safety, it is clear that an evaluative understanding of online communities and online security issues is necessary.

\section{DISCUSSION}

So far we have observed the way in which social networks behave in the physical world, which includes the way they form, evolve and support the students through the learning journey. However, as learning has evolved from a practice taking place in the physical world to computer-supported learning systems that mediate interaction with the learning material, establishing a strong foundation for substituting the social part of learning has become crucial. To date, many efforts have attempted to maintain the interaction of students in the on-line perspective through the use of social software. NJIT's virtual classroom [80] to certain extent is an evidence of success in this effort despite of many limitations, where student pursued their college degree while working full time anytime/anywhere interacting with their online peers, mentors and other students in the class while using social and advanced computer network. Recently a new generation of social software has the potential to deliver more effective support to users' social lives. This new generation of web-based software, known to web developers as "Web 2.0", has quickly gained widespread popularity, to the point that millions of users worldwide are creating content, tagging photographs, sharing videos, blogging, and making friends through the web every day. This perceived popularity has placed a new pressure upon universities competing within 'information economies' to acknowledge and apply social software effectively within education.

Social software has provided many features that can serve the learning sector in different ways. Multimedia, or any content on the web can be highly useful in tagging learning material for sharing between peers. This methodology, usually referred to as "the wisdom of the crowd", has been proposed [1] within social networks. Tagging can provide an easier way to obtain valuable experience from trusted members in the social network. Another area where social software offers value is within recommendations for learning material and sources of information. Recommender systems are usually based on similarities between the users. If users are from the same network and share similar interests, then it is easier and more reliable to apply recommendation algorithms for networks of learners rather than just individuals. In distance learning and virtual universities, the recommendation, tagging and sharing of resources and ideas can be highly beneficial given that students do not meet physically. The social value of face-to-face discussion can be partially replaced through the use of social software. Furthermore, if distance learners tend to be in the same network (university social network) and using social software for entertainment, this may result in their becoming more socially connected, thereby enhancing their social learning environment and student experience.

Rich internet applications enabling the next-generation web is another effort in this context. Microsoft Windows Live [59], Live Meeting [57] and Office Live [58] online services as well as other emerging new sets of tools, standards and development techniques such as AJAX (Asynchronous JavaScript and XML), are the most notably used technologies. These are all part of the Web 2.0 developments which are widely used in different social software such as Google Maps and Yahoo's Flicker photo sharing service for online communication by different networks.

The next generation of end-user programming tasks and environment with systems like Chickenfoot [11], which enables end-users to automate, customize, and integrate web applications without examining their source code by embedding a programming environment directly into the Firefox web browser, is another example of Web 2.0 application. It will be potentially used in the near future as a part of programming environments in learning for end user programmers and for the study of social networks by human factors researchers.

However, from our study, we also observe that existing software often ignores the significance of privacy, provenance, and trust.

As mentioned previously, in day-to-day life we rely on individuals within our social network based on the nature of the relationship that connects us with them. It is unusual for an individual to question the integrity of the others in their network or the provenance of the information within the network. However, social software is still in its infancy with regard to trust issues. Within education, authenticity and trust must be facilitated to enable confidence in personal security and the learning environment and social network. How can a stronger layer of trust enable social software to be used in learning in a more secure way? Furthermore, are there other factors that hold back social software from achieving a high success in the learning domain?

\section{FUTURE WORK}

In the future this research will investigate the impact of social networks on the student learning experience based on a survey involving students and lecturers. Our goal is to explore student learning experience when they are part of a social network. From the field questionnaires filled out by students and lecturers we aim to understand the perception of social networks as well as analyze its role and utility in learning. The survey will demonstrate:

- Guidelines for lecturers to apply social networks in course development to benefit student learning, and

- Guidelines for students to apply social networks to enhance their learning experience.

The results of the survey will assist the researchers in analyzing the use and efficiency of social networks in learning. Results will also indicate the possible use of social software 
to support social networks of learners on the web and elearning environments.

Key emergent questions will include: Is (student) knowledge acquisition aided by the processes through which social networks are formed? To what extent do existing technologies and frameworks associated with social networks facilitate learning behavior? What impact do group dynamics in a social network have on the learning process?

In an effort to evaluate the empirical application of our research, we propose to use a questionnaire given to both undergraduate and graduate students. After having gone through the questionnaire ourselves, we will ensure a broad sample by giving the questionnaire to students within the UK and EU member countries as well as the USA, ostensibly the countries of the working group participants. To be complete, but not necessarily as part of our final analysis, we will administer the questionnaire to willing lecturers and professors. The authors feel that this view point will provide a beneficial overview and contextual insight to the student's learning experience.

For our questionnaires we chose to apply a context-based approach to the discussion and analysis of significant themes. We identified several points to help us better understand the social network functioning, for instance, whether personal demographics and personal learning background have any effects on the network formation. Additionally, we aim to know more about the reasons students join or form a social network and what aspects of the learning experience are important to them. Some may like to work as a group, other individually, some face-to-face, others remotely.

In addition, we want to explore why students leave one or another network, maybe to go somewhere else, as well as why they stay. Is it on their own or due to an external decision?

With a broad range of questions, we should be able to have a clear understanding of the role social networks play in students' life and how it affects, or not, their learning experiences. Based on this analysis we aim to recommend what aspects of social network will be useful and should be accounted for student learning in the future.

\section{CONCLUSION}

This paper demonstrates the social dimensions of a collaborative learning network, its formation, its presence and its influence on different social networks in education. Also mentioned are the effects of a new generation of web-based software development, known as Web 2.0, in learning and social networks. The main contribution of the paper is to analyze current learning practices and find the connection between the learning practices and social networks. As this paper is written from a student's perspective, the analysis is intimately relevant to the student's learning experience.

The implications of this paper extend into both research and practice. More research needs to be conducted in the area of social learning presence, in both on-line and traditional educational environments. Research is also needed to determine the extent to which the perception of social presence influences student satisfaction, student motivation and other attitudinal factors. The same needs to be analyzed for students' actual cognitive and effective learning goals. From the instructors' perspective, research needs to be conducted to determine the effect of social presence in facilitating course design. Then comes the question of how social software can be effectively used to achieve these targets.

\section{ACKNOWLEDGEMENTS}

Our thanks go to Janet Carter for encouraging us to submit to a postgraduate working group session. We also would like to thank Quintin Gee and Su White for proof reading this paper and for their useful insights.

\section{REFERENCES}

[1] Al-Khalifa, H. S., and Davis, H. C. Harnessing the wisdom of crowds: How to semantically annotate web resource using folksonomies. In Proceedings of IADIS Web Applications and Research (WAR2006) (2006).

[2] Alani, H., Dasmahapatra, S., O'hara, K., and Shadbolt, N. Ontocopi - using ontology based network analysis to identify communities of practice. IEEE Intelligent Systems 18 (2003), 18-25.

[3] Andriessend, E., Soekijad, M., Veld, M. H. I. T., AND Poot, J. J. Dynamics of knowledge sharing communities. Tech. rep., Delft University of Technology, 2001.

[4] Angeli, C., Bonk, C., And Hara, N. Content analysis of online discussion in an applied educational psychology course. [On-line] (1998).

[5] Archee, R. Using computer-mediated communication in an educational context: Educational outcomes and pedagogical lessons of computer conferencing. The Electronic Journal of Communication 3, 2 (1993).

http://www.cios.org/getfile/ARCHEE_V3N293 [restricted access].

[6] Bales, R. F. Interaction Process Analysis. A Method for the Study of Small Groups. Cambridge, MA: Addison-Wesley, 1950/1951.

[7] Balsamo, A. Technologies of the Gendered Body: Reading Cyborg Women. Duke University Press, New York, December 1995.

[8] Bandura, A. Self-efficacy: Towards a unifying theory of behavioral change. Psychological Review 84 (1977), 191-215.

[9] Berglund, A., And Eckerdal, A. What do cs students strive to learn? Computer Science Education 16, 3 (2006), 185-195.

[10] Berkson, L. Problem-based learning: Have the expectations been met? Academic Medicine 68, 10 (1993), S79-S88.

[11] Bolin, M. End-User Programming for the Web. PhD thesis, MIT, http://groups.csail.mit.edu/uid/ projects/chickenfoot/mbolin-thesis.pdf, May 2005.

[12] Воотн, S. Learning computer science and engineering in context. Computer Science Education 11, 3 (2001), 169-188.

[13] BordiA, P. Face-to-face versus computer-mediated communication: A synthesis of the experimental 
literature. The Journal of Business Communication 34, 1 (1992), 99-120.

[14] Borgatta, E. F., And Bales, R. F. Interaction of individuals in reconstituted groups. Sociometry 16, 4 (1953), 302-320.

[15] Boulos, M., Maramba, I., And Wheeler, S. Wikis, blogs and podcasts: a new generation of web-based tools for virtual collaborative clinical practice and education. BMC Medical Education 6 , 41 (August 2006).

[16] Cayzer, S. Semantic blogging and decentralized knowledge management. Communications of the ACM 47, 12 (December 2004), 47-52.

[17] Chin, M. Facebook statistics. http://static.ak. facebook.com/press/facebook_statistics.pdf, May 2007.

[18] Citeulike. http://www.citeulike.org.

[19] Classmates. http://www.classmate.com.

[20] Cohoon, J. Recruiting and retaining women in undergraduate computing majors. SIGCSE Bulletin 34, 2 (2002), 48-52.

[21] Collison, C. Connecting the new organisation. how bp amoco encourages post-merger collaboration. Knowledge Management Review (2000).

[22] Cooper, J., And Weaver, K. Gender and Computers: Understanding the digital divide. Lawrence Erlbaum \& Associates, Mahwah, NJ, 2003.

[23] Dailymotion. http://www.dailymotion.com.

[24] del.icio.us. http://del.icio.us/.

[25] EnGEströM, Y. Learning by Expanding: an activity-theoretical approach to developmental research. Orienta-Konsultit: Helsinki., 1987.

[26] Facebook. http://www.facebook.com/.

[27] Filmtrust. http://trust.mindswap.org/FilmTrust/.

[28] Flickr. http://www.flickr.com/.

[29] Friendsreunited. http://www.friendsreunited.co.uk.

[30] Friendster. http://www.friendster.com/.

[31] Garrison, R., Anderson, T., And Archer, W. Critical inquiry in a text-based environment: Computer conferencing in higher education. Unpublished manuscript, 2000.

[32] Goecks, J., And Cosley, D. Nuggetmine: Intelligent groupware for opportunistically sharing information nuggets. In Proceedings of the 2002 International Conference on Intelligent User Interfaces (IUI 2002) (2002), ACM, pp. 87-94.

[33] Goecks, J., And Mynatt, E. Leveraging social networks for information sharing. Computer Supported Cooperative Work 04 6, 3 (2004), 328-331.

[34] Gunawardena, C., And Zittle, F. Social presence as a predictor of satisfaction within a computer mediated conferencing environment. American Journal of Distance Education, 11, 3 (1997), 8-26.

[35] Hamasaki, M., And Takeda, H. Find better friends? re-configuration of personal networks by neighborhood matchmaker method. In $S W A F T$ (2003), pp. 73-76.

[36] Harasim, L. Shift happens: online education as a new paradigm in learning. Internet and Higher Education 3 (2000), 41-61.
[37] Heath, C., And Heath, D. Finding just enough of that sticky stuff. Brandweek 48, 1 (2007), 21-25.

[38] JACKSON, M. O. A survey of models of network formation: Stability and efficiency. Group Formation in Economics: Networks, Clubs, and Coalitions (2003).

[39] Johnson, D. W., And Johnson, F. P. Joining Together. Group Theory and Group Skills. Boston: Allyn and Bacon, 1975/1997.

[40] Jordan, M. H., Feild, H. S., And Armenakis, A. A. The relationship of group process variables and team performance. a team-level analysis in a field setting. Small Group Research 33, 1 (2002), 121-150.

[41] Kinnunen, P., And Malmi, L. Problems in problem-based learning - experiences, analysis and lessons learned on an introductory programming course. Informatics in Education 4, 2 (2005), 193-215.

[42] Kinnunen, P., and Malmi, L. Why students drop out cs1 courses? In Proceedings of ICER 2006 (2006), ACM, pp. 97-108.

[43] Kinnunen, P., McCartney, R., Murphy, L., And Thomas, L. Through the eyes of instructors: a phenomenographic investigation of student success. In Proceedings of ICER (2007).

[44] Kuenn, T. Communication innovation on a bbs: A content analysis. Interpersonal Computing and Technology: An Electronic Journal for the 21st Century 1, 2 (1993).

[45] Kuutti, K. Context and Consciousness: Activity Theory and Human Computer Interaction. Cambridge MIT Press, 1995, ch. Activity Theory as potential framework for human-computer interaction research, pp. 17-44.

[46] Lastfm. http://www.lastfm.com/.

[47] LAURILlard, D. Rethinking University Teaching 2nd Edition. Routledge-Falmer, London, 2002.

[48] Lave, J., And Wenger, E. Situated learning: Legitimate peripheral participation. 1-38, Palo Alto, CA, Institute for Research Learning, 1990.

[49] Leuf, B., And Cunningham, W. The Wiki Way. Addison Wesley, Boston, MA, 2001.

[50] Margolis, J., AND Fisher, A. Unlocking the clubhouse: women in computing. The MIT Press, 2003.

[51] Marton, F., Dall'Alba, G., And Beaty, E. Conceptions of learning. International journal of educational research 19, 3 (1993), 277.

[52] Maybury, M., D’Amore, R., And House, D. Expert finding for collaborative virtual environments. Communications of the ACM 44, 12 (December 2001), 55-56.

[53] Mayfield, R. Social Network Dynamics and Participatory Politics. O'Reilly, Lulu.com, 2005, ch. 10, pp. 116-132.

[54] McDonald, D. W., And Ackerman, M. S. Expertise recommender: A flexible recommendation sytem and architecture. In Computer Supported Cooperative Work 2000 (2000), ACM, pp. 231-240.

[55] McPherson, M., Smith-Lovin, L., And Cook, J. M. Birds of a feather: Homophily in social 
networks. Annual Review of Sociology 27 (2001), 415-44.

[56] Mehrabian, A. Some referents and measures of nonverbal behavior. Behavior Research Methods and Instrumentation 1, 6 (1969), 205-207.

[57] Microsoft live meeting. http://office.microsoft . com/en-us/livemeeting/default.aspx.

[58] Microsoft office live. http://www . officelive.com.

[59] Microsoft windows live. http://get.live.com/.

[60] Morales-Mann, E. T., and Kaitell, C. A. Problem-based learning in a new canadian curriculum. Journal of Advanced Nursing 33, 1 (2001), 13-19.

[61] Myspace. http://www.myspace.com/.

[62] Nathan, R. My Freshman Year: What a Professor learned by becoming a student. Cornell University Press, Ithaca, NY, August 2005.

[63] Nuutila, E., Törmä, S., And Malmi, L. Pbl and computer programming - the seven steps method with adaptations. Computer Science Education 15, 2 (2005), 123-142.

[64] Orkut. http://www.orkut.com/.

[65] Ounnas, A., Davis, H. C., And Millard, D. E. Towards semantic group formation. In The 7th IEEE International Conference on Advanced Learning Technologies (Niigata, Japan, July 2007), ICALT 2007. (in press).

[66] Ounnas, A., Liccardi, I., Davis, H. C., Millard, D., AND White, S. A. Towards a semantic modeling of learners for social networks. In $S W$-EL (2006), Workshop at the AH 06, pp. 1-6. Dublin, Ireland

[67] Preece, J. Online Communities - Designing Usability, Supporting Sociability. John Wiley and Sons Ltd, 2000.

[68] Prensky, M. Digital natives digital immigrants. On the Horizon, NCB University Press 9, 5 (October 2001).

[69] Prentice, D. A., Miller, D. T., and Lightdale, J. R. Asymmetries in attachments to groups and their members: Distinguishing between common-identity and common-bond groups. Personality and Social Psychology Bulletin 20, 5 (1994), 484-493.

[70] Reunion. http://www.reunion.com/.

[71] Rohde, M., Klamma, R., Jarke, M., and Wulf, $\mathrm{V}$. Reality is our laboratory: communities of practice in applied computer science. Behaviour $\mathcal{E}$ Information Technology 26, 1 (January 2007), 81-94.

[72] Rourke, L., Anderson, T., Garrison, D. R., AND Archer, W. Assessing social presence in asynchronous text-based computer conferencing. Journal of Distance Education/Revue de l'enseignement à distance (2001).

[73] SALjo, R. Learning in educational settings: Methods of inquiry. P. Ramsden (Ed.), Improving Learning: New Perspectives (1988), 32-48, New York: Kogan Page.

[74] Sassenburg, K. Common bond and common identity groups on the internet: Attachment and normative behavior in on-topic and off-topic chats.
Group Dynamics: Theory, Research, and Practice 6, 2 (2002), 27-37.

[75] Schellens, T., Van Keer, H., Valcke, M., And De Wever, B. Learning in asynchronous discussion groups: a multilevel approach to study the influence of student, group and task characteristics. Behavior and Information Technology 26, 1 (January 2007), $55-71$.

[76] Schmidt, H. G. Problem-based learning: rationale and description. Medical Education 17, 1 (1983), $11-16$.

[77] Schwen, T. M., ANd Hara, N. Community of practice: A metaphor for online design? The Information Society 19 (2003), 257-270.

[78] Second life. http://www. secondlife.com/.

[79] Slattery, K. L. Loyalty, harm and duty: Pbl in a media ethics course. Public Relations Review 28 (2002), 185-190.

[80] S.R.Roxanne, and B.Wellman. Asynchronous learning networks as a virtual classroom. Communications of the ACM 40, 9 (September 1997), 44-49.

[81] Tajfel, H. Social Identity and Intergroup Relations. Cambridge University Press, Cambridge, 1982.

[82] Tinto, V. Classrooms as communities: Exploring the educational character of student persistence. Journal of Higher Education 68, 6 (Nov. - Dec. 1997), 599-623.

[83] Tinto, V. Learning better together: The impact of learning communities on student success in higher education. Journal of Institutional Research 9, 1 (2000), 48-53.

[84] Tuckman, B. W. Developmental sequence in small groups. Psychological Bulletin 63, 6 (1965), 384-399.

[85] Veoh. http://www.veoh.com/.

[86] Vivacqua, A., And Lieberman, H. Agents to assist in finding help. In CHI (2000), ACM, pp. 65-72.

[87] Vygotsky, L. S. Mind in Society. Cambridge: Harvard University Press, 1978.

[88] WAKEForD, N. The embedding of local culture in global communication: independent internet cafes in london. New Media and Society 5, 3 (2003), 379-399.

[89] Walther, J. B. Computer-mediated communication: Impersonal, interpersonal, and hyper-personal interaction. Communication Research 23, 1 (1996), 3-43.

[90] Wegerif, R. The social dimension of asynchronous learning networks. Journal of ALN 2 (1998), 34-49.

[91] Wellman, B. Physical place and cyberplace: The rise of personalized networking. International Journal of Urban and Regional Research 25, Special Issue on "Networks, Class and Place" (2001). Edited by Talja Blokland and Mike Savage.

[92] Wenger, E. Communities of practice: Learning, meaning and identity. Cambridge University Press (1998).

[93] Wenger, E., And Snyder, W. M. Communities of practice: The organizational frontier. Harvard Business Review (2000).

[94] Wheelan, S. A., and Williams, T. Mapping 
dynamic interaction patterns in work groups. Small Group Research 34, 4 (2003), 443-467.

[95] Wiki choicetree.

http://c2.com/cgi/wiki?WikiChoicetree, May 2005.

[96] Wikipedia. http://www.wikipedia.org/, May 2005.

[97] Winter, M. Developing a group model for student software engineering teams. Tech. rep., Department of Computer Science. Saskatoon, Saskatchewan, University of Saskatchewan, Canada, 2004.

[98] Yang, F., Han, P., Shen, R., Kraemer, B. J., AND FAN, X. Cooperative learning in self-organizing e-learner communities based on a multi-agents mechanism. Lecture Notes in Artificial Intelligence 03 1 (2003), 490-500.

[99] Yee, S., And Park, K. Studiobridge: Using group, location, and event information to bridge online and offline encounters for co-located learning groups. In Proceedings of the SIGCHI conference on Human factors in computing systems (2005), ACM Press, pp. 551-560.

[100] Youtube. http://www youtube.com/.

[101] Zhang, J., And Ackerman, M. S. Searching for expertise in social networks: a simulation of potential strategies. In GROUP (2005), ACM, pp. 71-80. 